\title{
Error Estimates of the Ares I Computed Turbulent Ascent Longitudinal Aerodynamic Analysis
}

\author{
Khaled S. Abdol-Hamid ${ }^{*}$ and Farhad Ghaffari* \\ NASA Langley Research Center \\ Hampton, Virginia
}

Numerical predictions of the longitudinal aerodynamic characteristics for the Ares I class of vehicles, along with the associated error estimate derived from an iterative convergence grid refinement, are presented. Computational results are based on an unstructured grid, Reynolds-averaged Navier-Stokes analysis. The validity of the approach to compute the associated error estimates, derived from a base grid to an extrapolated infinite-size grid, was first demonstrated on a sub-scaled wind tunnel model at representative ascent flow conditions for which the experimental data existed. Such analysis at the transonic flow conditions revealed a maximum deviation of about $23 \%$ between the computed longitudinal aerodynamic coefficients with the base grid and the measured data across the entire roll angles. This maximum deviation from the wind tunnel data was associated with the computed normal force coefficient at the transonic flow condition and was reduced to approximately $16 \%$ based on the infinite-size grid. However, all the computed aerodynamic coefficients with the base grid at the supersonic flow conditions showed a maximum deviation of only about $\pm 8 \%$ with that level being improved to approximately $\pm 5 \%$ for the infinite-size grid. The results and the error estimates based on the established procedure are also presented for the flight flow conditions.

\section{Nomenclature}

$\begin{array}{lll}C_{A F} & = & \text { axial force coefficient, } F_{X} / q_{\infty} S_{r e f} \\ C_{N} & = & \text { normal force coefficient, } F_{Z} / q_{\infty} S_{r e f} \\ C_{m} & = & \text { pitching moment coefficient, pitching moment } / q_{\infty} S_{r e f} D \\ C_{R M} & = & \text { rolling moment coefficient, rolling moment } / q_{\infty} S_{r e f} D \\ C P & = & \text { center of pressure, } C_{m} / C_{N} \\ D & = & \text { diameter, ft } \\ F_{X} & = & \text { axial force, lbf } \\ F_{Z} & = & \text { normal force, lbf } \\ h r & = & \text { hour } \\ M & = & \text { Mach number } \\ N & = & \text { total number of cells; used for grid parameter estimation } \\ q_{\infty} & = & \text { free-stream dynamic pressure, psf } \\ R e & = & \text { Reynolds number based on D } \\ S_{r e f} & = & \text { reference area, ft }{ }^{2} \\ y^{+} & = & \text {inner law distance } \\ \alpha & = & \text { angle of attack, degrees } \\ \Delta & = & \text { incremental value } \\ \phi & = & \text { vehicle roll angle, also Phi, degrees }\end{array}$

\section{Introduction}

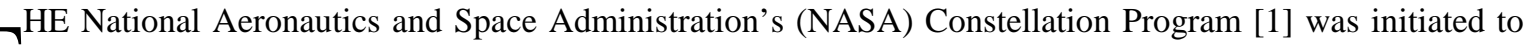
1 address the needs of U.S. access to space in the post Space Shuttle era with proposed missions to the Moon, Mars and beyond. Unlike the Space Shuttle, where both crew and cargo are launched simultaneously, the architecture for the Constellation Program proposed two separate launch vehicles: the Ares I for the crew and the Ares V for the cargo. The Ares I, the vehicle of interest in the present study, was a two-stage rocket that incorporated a modified five-segmented solid rocket booster (SRB) from the Shuttle program while the upper stage was to be powered by a redesigned J-2X engine from the Apollo Program. Figure 1 shows, conceptually, the major components of the vehicle. The Ares I major components consist of the launch abort system (LAS),

* Aerospace Engineer, Configuration Aerodynamic Branch, Associate Fellow AIAA 
crew exploration vehicle (CEV) named Orion, the upper stage (US), and the first stage (FS) SRB that includes the frustum and the aft skirt.

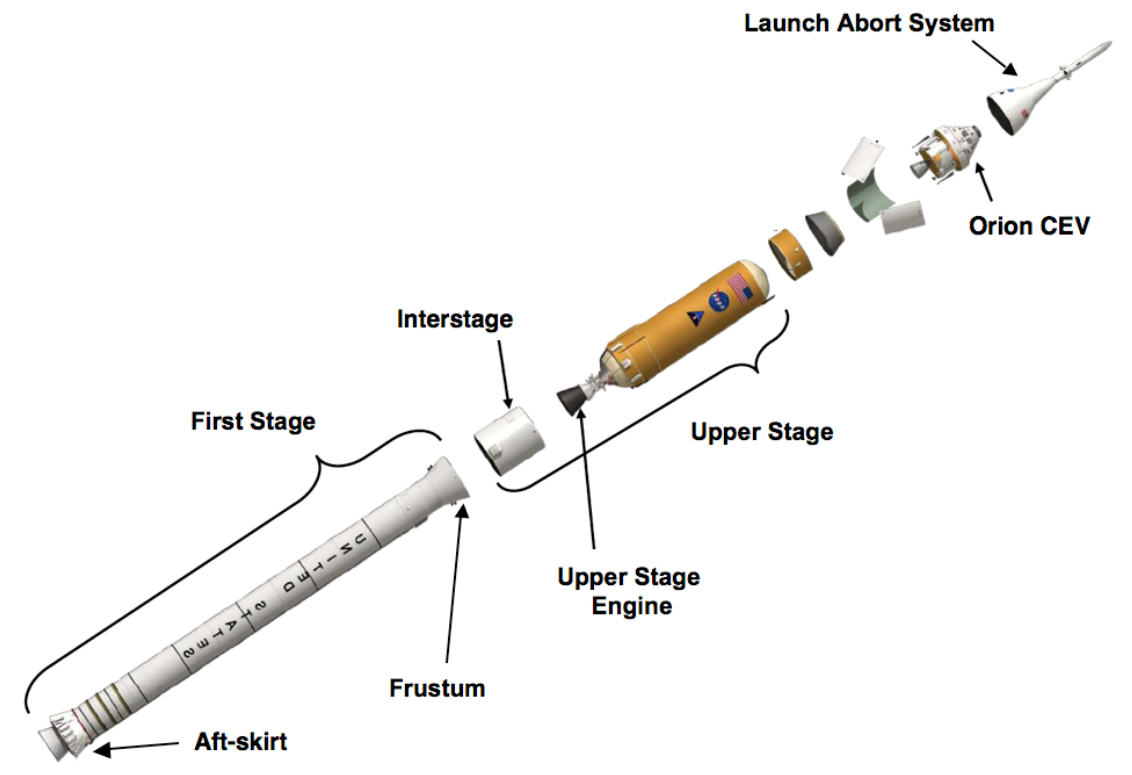

Figure 1. Ares I major components.

Considered complementary to ground based wind tunnel testing and flight tests, computational fluid dynamic (CFD) methods were extensively used to aid the development of the various aerodynamic databases for the proposed crew launch vehicle. One of the CFD tools utilized was the NASA Langley Research Center (LaRC) developed Navier-Stokes flow solver USM3D [2]. USM3D had the primary role within the Ares project to develop the necessary computational ascent aerodynamic data while other widely used flow solvers, FUN3D [3] and OVERFLOW [4], were to have supporting roles to provide complementary results for fewer cases as part of a confidence building process to ensure code-to-code solution consistency. The free-stream flow conditions considered typical for the nominal ascent flight trajectory generally ranged over $0^{\circ}<\alpha<8^{\circ}, 0.5<M$ $<4.5$, and at various vehicle roll angles $(\phi)$ over 360 degrees.

An overview of the prior computational results and analyses along with the processes used to assess, verify, and validate the numerical aerodynamic predictions is presented in reference [5] for the evolving Ares I design analysis cycle (ADAC). As discussed in the reference, the USM3D results were verified against those obtained from other Navier-Stokes flow solvers, whereas the validations were assessed through comparisons with the available wind tunnel data. Only limited sets of studies were conducted primarily at a benchmark system level (i.e., clean configuration with no protuberances) during the course of the prior computational activities to address solution sensitivities to grid refinement. As a result, the objective of the present study is to provide a systematic approach to tackle the issue by providing the estimates of the computational error in the predicted longitudinal aerodynamic coefficients of the ADAC-3 (A106) configuration at a system level (i.e., vehicle with full protuberances). The Ares I vehicle contained numerous protuberances, adding to the complexity of the geometry and the resulting flow physics both from the numerical simulation aspect as well as the wind tunnel model design, manufacturing, and testing. It is important to note that, the present error estimate study utilized the prior base grid [5], used to generate the ADAC-3 (A106) longitudinal aerodynamic coefficients, as the medium grid level to complement the coarse and fine grid levels. The computations were performed at the nominal ascent flight trajectory and without including any propulsion effects. The initial analyses were conducted at wind tunnel flow conditions for which the appropriate experimental data were available to gauge the validity of the numerical predictions and the associated error estimates. Once established, the procedures were then applied to compute the aerodynamic estimates at representative flight Reynolds number flow conditions for which no experimental data existed. In addition, the present analysis includes the relevant data to distinguish the balance between the prediction accuracy against the grid size and the corresponding computer 
resource requirements for the computations at both wind tunnel and flight flow conditions. The present analyses were performed only at the selected flow conditions of $\alpha=8^{\circ}, M=0.9,1.6,3.0$ which is a subset of the conditions used in the prior computations [5]. These computations were performed at nominal roll angles $(\phi)$ ranging from $0^{\circ}$ to $360^{\circ}$ at $30^{\circ}$ increment. The detailed analysis presented in this paper only focuses on the numerical data obtained at $\alpha=8^{\circ}, M=1.6$ flow conditions, which is considered to be near the maximum dynamic pressure that the vehicle would experience during the ascent trajectory. However, a summary of the final results that encompasses the entire computational matrix is presented for completeness.

\section{Computational Approach}

\section{USM3D Flow Solver}

USM3D is a tetrahedral cell-centered, finite volume, Euler and Navier-Stokes flow solver. Time integration follows the implicit point Gauss-Seidel algorithm, explicit Runge-Kutta approach, and local time stepping for convergence acceleration. The solver scheme allows various options for computing the inviscid flux quantities across each cell face. These include Roe's flux-difference splitting (FDS), advection upstream splitting method (AUSM), flux vector splitting (FVS), and the Harten, Lax, and van Leer with contact restoration (HLLC) scheme. MinMod and Superbee flux limiters have been incorporated in the flow solver to smooth out the flow discontinuities due to shock waves. Turbulence models include Spalart-Allmaras (SA) [6] with and without the wall function, $k-\varepsilon$ [7], shear stress transport (SST) [8], and algebraic stress models. In addition to the standard slip/no-flow through (inviscid surface) and the no-slip/no-flow through (viscous surface) boundary conditions, the method also includes a transpiration boundary condition that can be used to simulate propulsion effects.

\section{Grid Generation}

The surface triangulations along with the field tetrahedral volume grids were generated using the GRIDTOOL [9] and VGRID [10] software developed at LaRC. A rectangular box that encompasses the vehicle is typically used to define the computational domain far-field boundaries. Each face of this rectangular box is located several body lengths away from the configuration in the upstream, radial and downstream direction. All computational grids used in the present analysis included a sting geometry representation of the Wind Tunnel (WT) model. The detailed grid description used for the computations at WT Re and Flight (FLT) Re will be discussed later. As a general practice, each final converged solution is analyzed to insure that the turbulent sublayer, has been grid resolved and that the average $y^{+}$is less than 1 .

\section{Solution development and convergence}

Many efforts were made during the previously conducted research [5] to establish the appropriate parameter settings for the USM3D flow solver, such as the flux schemes, limiters, and turbulence model to ensure the solution development remained stable over the nominal range of ascent flow conditions [5]. This led to the development of an efficient script that would automatically generate appropriate input-stream parameters to the flow solver and submit a series of cases such as a Mach number or angle of attack sweep to a designated computer platform. Upon completion, a summary page pertinent to the solution convergence characteristics would be generated for the validity assessments. This summary page includes the designed statistical convergence data for the longitudinal and rolling moment coefficients as well as the flow solution residuals.

Solution convergence was evaluated by monitoring the overall fluctuations in all six force and moment coefficients. In particular, a solution was considered iteratively converged when the fluctuations in $C_{N}, C_{A F}, C_{m}$, and $C_{R M}$ become generally less than $0.5 \%$ of its average final value over the last 2000 iterations and an appropriate level of reduction in the mean flow residual for density and turbulent model residual. As a result, the error estimate due to iterative solution convergence was considered very small and therefore is not included in the present analysis. All present computations were based on the SA turbulence model and have been performed on the Pleiades computer system at the NASA Advanced Supercomputer (NAS) complex located at the Ames Research Center. In the present study, the variations in Mach and Reynolds number, grid levels, and the vehicle's roll angle produced a total of 216 cases for which the solution generation required about 1,500,000 hours of Central Processing Unit (CPU) time.

\section{Results and Discussions}

\section{Computations at wind tunnel Re}


The guidelines established by the Drag Prediction Workshop [11] and the High Lift Prediction Workshop [12] to address solution sensitivity to grid refinement were adopted for estimating the computational error in the previously computed longitudinal aerodynamic coefficients [5]. The results obtained from the base grid (BG) in the prior computations provided the reference data for the present analysis and, in particular, for generating the properly sized coarse and fine grid levels. Figure 2 shows the representative computational grids on the surface and the near-field view of a typical cross-sectional cut for the coarse, base, and fine grid. There were roughly over 40 protuberances, with different sizes and shapes, which were distributed nonuniformly over the configuration. Such geometrical complexity presented a challenge for the grid generation and the flow solver to properly capture the associated flow physics and the resulting aerodynamic properties of the vehicle across a fairly wide range of flow conditions. Approximately $35 \%$ of the total number of surface elements for the vehicle was used to represent these major protuberances.
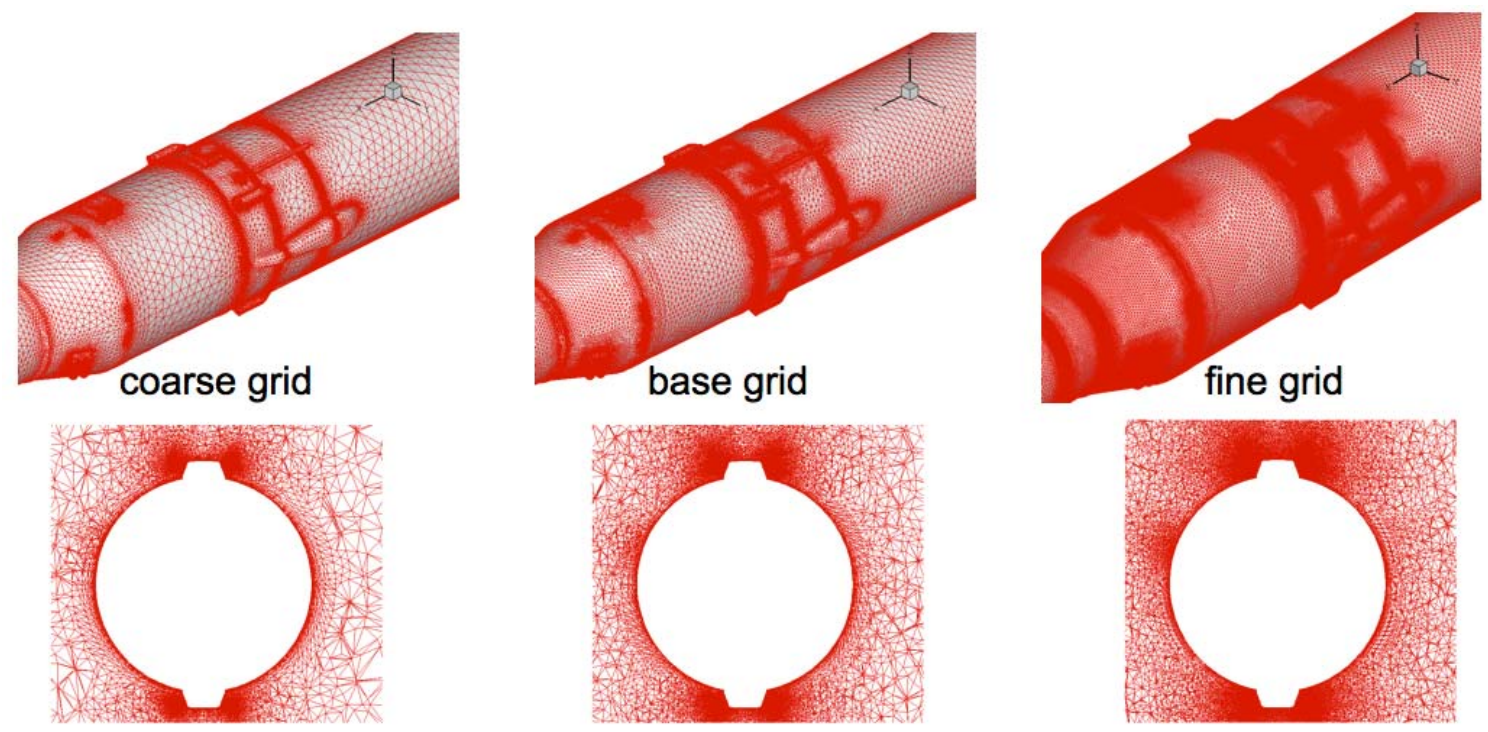

Figure 2. Computational grid distribution on the surface and a typical cross-flow plane for the coarse, base, and fine grids for the ADAC-3 (A106) configuration.

Relative to the base grid (BG), a factor of three was used to size the corresponding number of cells in the coarse and fine grid as shown in Table 1. Based on this approach, the coarse and the fine grid consisted of onethird and three times the number of cells contained in the BG, respectively. These ratios were maintained to be approximately the same in both the viscous boundary layer region as well as in the inviscid field. Furthermore, a factor of 1.26 was used to construct the viscous layers $\left(\delta_{\mathrm{j}}\right)$ in the radial direction based on the following equation from Ref. 11 by setting the grid growth rate parameters $R 1=0.16$ and $R 2=0.04$ for all the grids used in the present investigation.

$$
\delta_{j}=\delta_{1}\left(1+R 1(1+R 2)^{j-1}\right)^{j-1}
$$

In this equation, the variable $\delta_{1}$ is the first cell height next to the solid surface in the normal direction.

The computational domain far-field boundaries were chosen to be at about 100 SRB diameters away from the vehicle. Table 1 also provides the values of $\delta_{1}$, the total number of cells in the viscous layer and the total number of surface elements for different grids. The factor of 2/3 was also used to size the value of $\delta_{1}$ for each consecutive grid refinement. In addition, Table 1 presents the approximate range for the number of iterations along with the computer resource requirements (i.e., CPU-hr range) for a typical case. In an effort to improve the grid quality, a grid optimizer [13] was employed to smooth out any local grid skewness or a large variation of the cell volumes prior to the launching of the flow solver. This activity was shown to stabilize and enhance the solution convergence characteristics over the examined range of flow conditions. In addition, as shown in Table 1, there was roughly the expected linear relationship between the total number cells $(\mathrm{N})$ and the corresponding computer resource requirements. As a result, the fine grid computations required approximately 
three times the CPU hours to converge the solution as those needed for the BG. This similarity in computer usage remained approximately the same between the base and the coarse grid.

Table 1 ADAC-3 (A106) grid statistics and computer resource requirements for WT Re cases

\begin{tabular}{|c|c|c|c|c|c|c|}
\hline Grid & $\begin{array}{c}\text { Number of } \\
\text { cells, } \boldsymbol{N}\end{array}$ & $\begin{array}{c}\mathbf{1}^{\text {st }} \text { cell height, } \\
\boldsymbol{\delta} \text {, inches }\end{array}$ & $\begin{array}{c}\text { Number of } \\
\text { viscous cells }\end{array}$ & $\begin{array}{c}\text { Number of } \\
\text { surface } \\
\text { triangles }\end{array}$ & \#Iterations & $\begin{array}{c}\text { Computer } \\
\text { Resources } \\
\text { CPU-hr range }\end{array}$ \\
\hline coarse & $22,502,721$ & 0.0225 & $17,713,428$ & 377,272 & $15,000-25,000$ & $600-1000$ \\
\hline base & $70,203,102$ & 0.015 & $46,827,963$ & $1,023,590$ & $15,000-25,000$ & $2,000-4,000$ \\
\hline fine & $214,373,376$ & 0.01 & $115,818,453$ & $2,568,446$ & $15,000-25,000$ & $6,000-10,000$ \\
\hline
\end{tabular}

The coarse, base, and the fine grids were used to compute the flow at $\alpha=8^{\circ}, M=0.9,1.6,3.0$, and appropriate wind tunnel $R e$. These computations were performed at nominal roll angles $(\phi)$ ranging from $0^{\circ}$ to $360^{\circ}$ at $30^{\circ}$ increment. Figure 3 shows the effects of the grid refinement on the computed $C_{A F}$ and $C_{N}$ at the representative flow conditions of $\alpha=8^{\circ}$ and $M=1.6$ as a function of grid parameter $\left(N^{-2 / 3}\right)$ for various roll angles. The results for both $C_{A F}$ and $C_{N}$ show a roughly second-order asymptotic behavior reaching a limiting value for an assumed infinite-size grid (IG) as the grid parameter approaches zero over the vertical axis. It should be noted that the grid parameter increment $\left(\Delta N^{-2 / 3}\right)$ between the base and the fine grid was approximately the same as that of the fine and the IG. This feature was indicative of the appropriateness on the total number of cell size selections between the base and the fine grid that resulted in roughly a linear extrapolation to obtain the IG values. Similarly, Figure 4 shows the procedure used for extrapolating the computed $C_{A F}$ at various roll angles to determine the limiting value for an IG. The top portion of the figure shows the computed $C_{A F}$ for various roll angles and the extrapolation step for two selected roll angles of $30^{\circ}$ and $240^{\circ}$. The lower left portion of the figure shows the limiting values of the coefficient, determined from an extrapolation of the results from the BG to an IG by an error bar-lines for all computed roll angles. The lower right portion of the figure shows the percent variation of the computed $C_{A F}$ for the limiting values from the BG to be less than about $4 \%$ for all the roll angles and that the maximum occurs at $\phi=30^{\circ}$.

A similar procedure was employed to determine the limiting values of the computed $C_{N}$ for various roll angles with the results shown in Figure 5. Analogous to the $C_{A F}$, the computed $C_{N}$ also showed a monotonic behavior with grid refinement. The percent change of the $C_{N}$ limiting values were shown to be less than $10 \%$ for all the roll angles and that the maximum occurred at $\phi=300^{\circ}$.
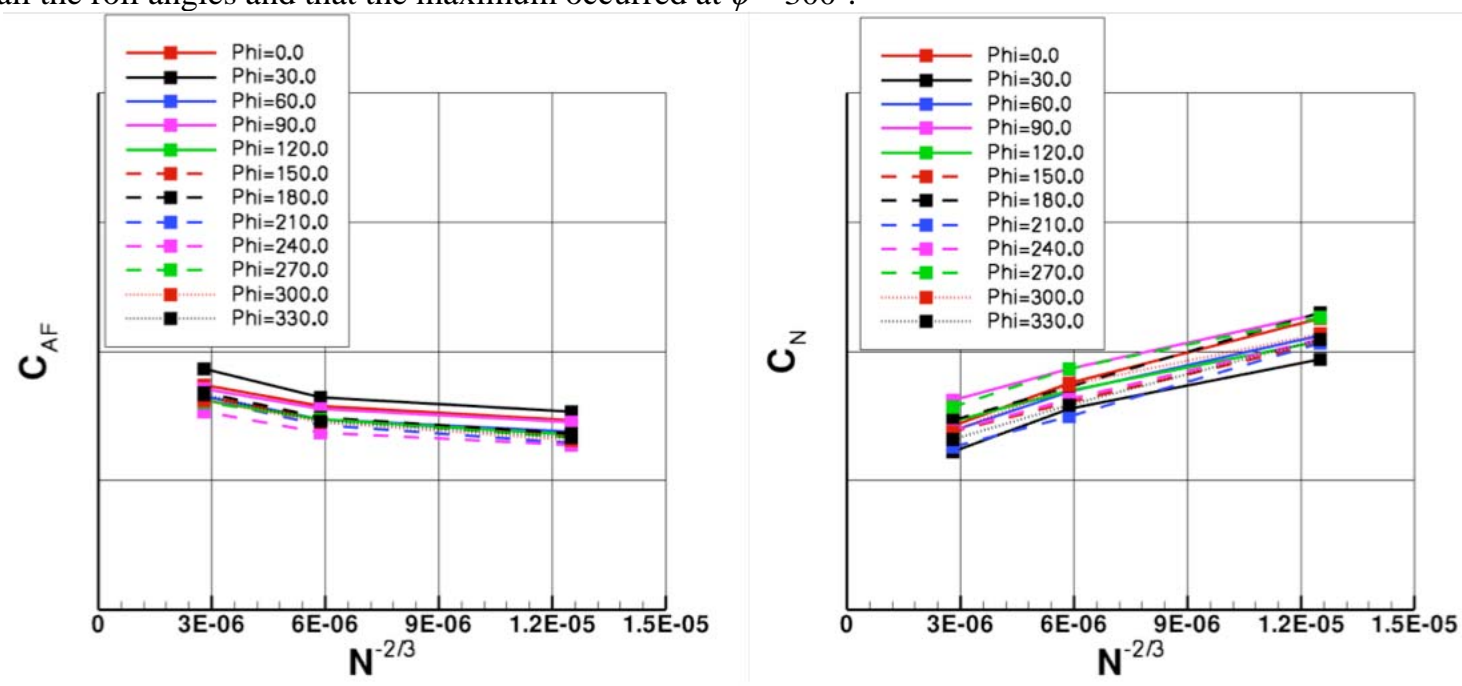

Figure 3. Effects of grid refinement on computed $C_{A F}$ and $C_{N}$ at various roll angles, $M=1.6, \alpha=$ $8^{0}$, WT Re.

5

American Institute of Aeronautics and Astronautics 

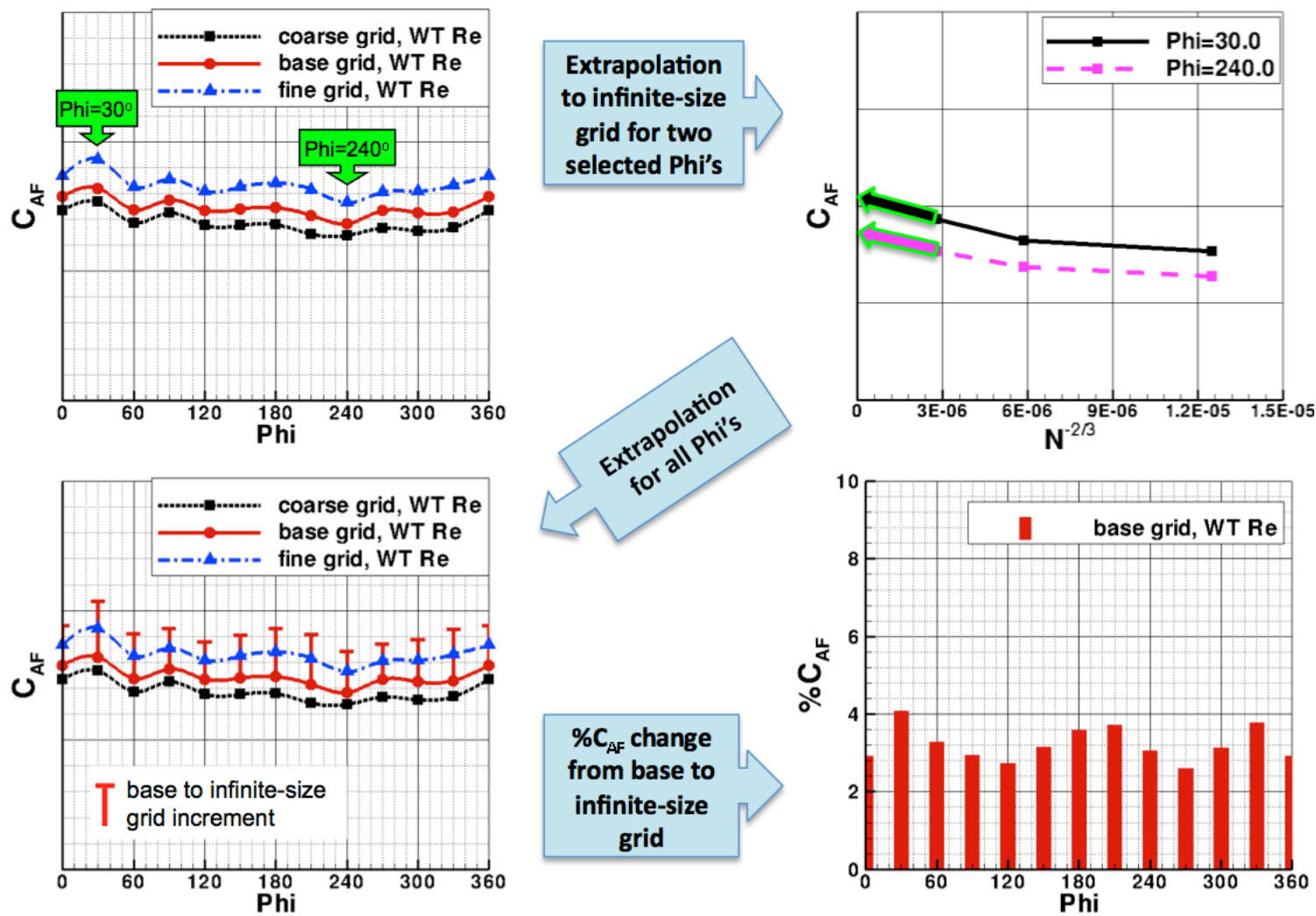

Figure 4. Extrapolation procedure to determine the $C_{A F}$ for an infinite-size grid and the percent error estimates from the base grid results, $M=1.6, \alpha=8^{0}$, WT $R e$.
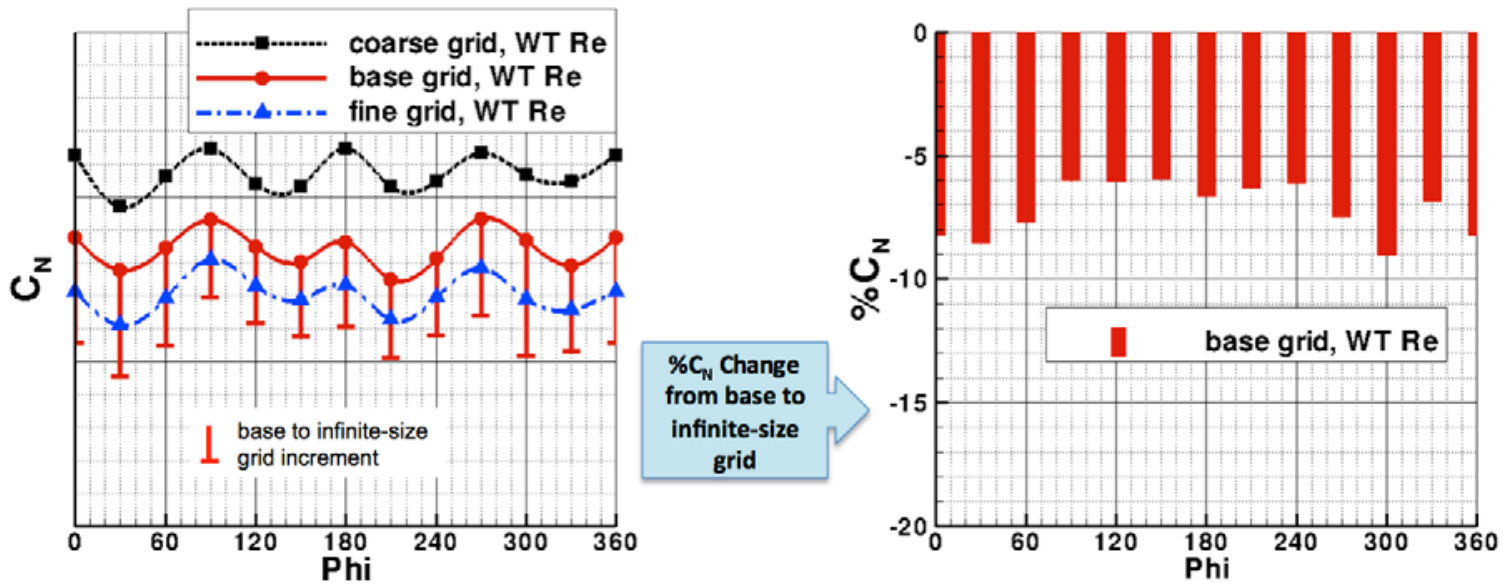

Figure 5. Effects of grid refinement on computed $C_{N}$ and the corresponding percent error estimates from base grid to an infinite-size grid, $M=1.6, \alpha=8^{\circ}$, WT Re.

Figure 6 shows the effects of iterative convergence grid refinement on the computed longitudinal aerodynamic coefficients as well as the center of pressure for the examined Mach numbers at WT Re. These data were the maximum error bar-lines or the limiting values of the extrapolated results from the base to the infinite-size grid across the entire range of roll angle. The results generally indicated a less than $\pm 10 \%$ variation in the computed coefficients due to the grid refinement over the examined flow conditions. In addition, the results also showed that the largest grid dependency occurred at transonic and low supersonic flow conditions for the computed $C_{N}$ and $C_{m}$ and that the overall variations decreased dramatically to less than $\pm 3 \%$ at the higher Mach number of 3. 


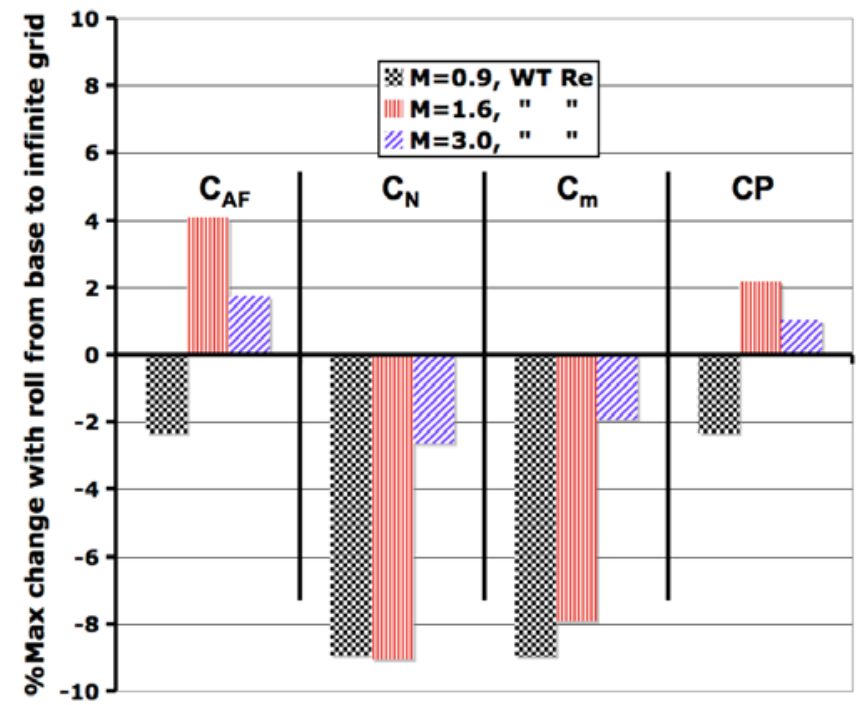

Figure 6. Percent maximum error estimate with roll angle in the computed longitudinal aerodynamic characteristics and the center of pressure from the base to an infinite-size grid for various Mach numbers at $\alpha=8^{\circ}$, WT Re.

The computed $C_{A F}$ results from the base grid and the extrapolated values for the infinite-size grid (shown with the error bar-lines) were correlated with the available experimental data in Figure 7 for various roll angles at $M=1.6, \alpha=8^{\circ}$. The experimental data were obtained from the LaRC Unitary Plan Wind Tunnel (UPWT) [14] and the Boeing Polysonic Wind Tunnel (PSWT) [15]. These experimental results, developed within the Ares Project, have been corrected for the tunnel flow angularity and model balance/sting aeroelastic deflections. In addition, the figure shows the experimental uncertainty error maximum percent value due to wind tunnel data repeatability [see figures 1-3 of Reference 16] for the examined Mach numbers of 0.9, 1.6 as well as 3.0. The results, shown on the left side of the figure, indicate that the BG clearly underpredict the measured $C_{A F}$ across the roll angles; however, an improved correlation has been achieved between the IG and the wind tunnel data. As shown on the right side of the figure, the percent deviation between the computed $C_{A F}$ from the $B G$ and the average values between the two wind tunnel data were less that $5 \%$ whereas it was less than $\pm 1 \%$ for the IG across the examined roll angles. Such a percent deviation in the latter computed $\mathrm{C}_{\mathrm{AF}}$ from the measured data is well within the associated experimental uncertainty error.
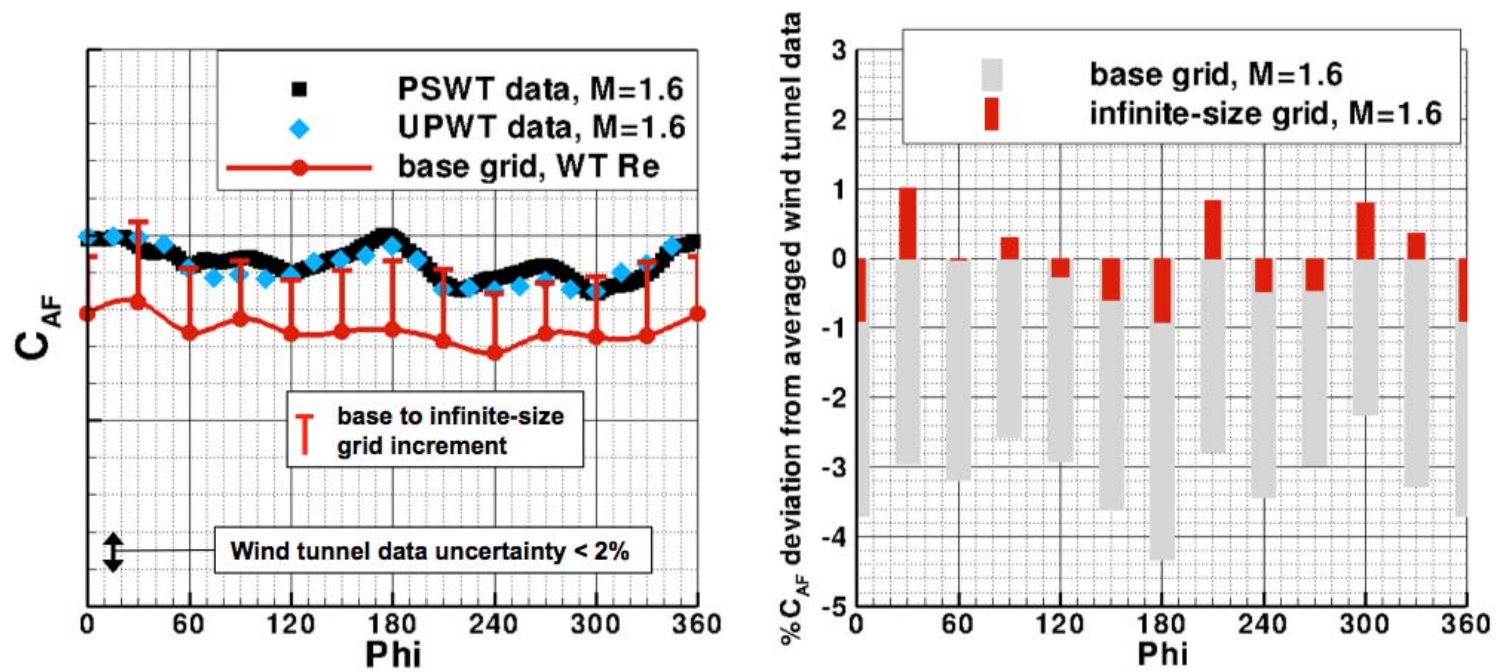

Figure 7. Computed $C_{A F}$ from the base and infinite-size grid and correlation with wind-tunnel data along with their percent deviation, $M=1.6, \alpha=8^{\circ}$.

7

American Institute of Aeronautics and Astronautics 
Similarly, the computed $C_{N}$ results obtained from the BG and the extrapolated values for the IG were correlated with the measured wind tunnel data in Figure 8 for various roll angles. The results, shown on the left side, indicate an overprediction of the computed $C_{N}$ using the BG at all examined roll angles, while an improved overall correlation has been achieved between the IG predictions and the average values of measured data from the two wind tunnels. As shown on the right side of Figure 8, such an improved correlation was within a 5\% deviation as opposed to about $8 \%$ deviation for the computed results with the BG from the averaged measured data. With the exception of few roll angles (i.e., Phi $=0^{\circ}, 180^{\circ}, 240^{\circ}$ and $270^{\circ}$ ), the percent deviation of the computed $\mathrm{C}_{\mathrm{N}}$, for the infinite-size grid, from the averaged wind tunnel data is well within the uncertainty error due to data repeatability. Although not shown here, it should be noted that such an improved correlation in the computed $C_{A F}$ and $C_{N}$, between the IG and experimental data, was not relatively as well behaved for the pitching moment coefficient at a few roll angles despite the presence of a monotonic trend. However, the percent deviation of the computed $C_{m}$, using the BG and the IG, from the averaged wind tunnel data still remained well within $8 \%$ across the examined roll angles.
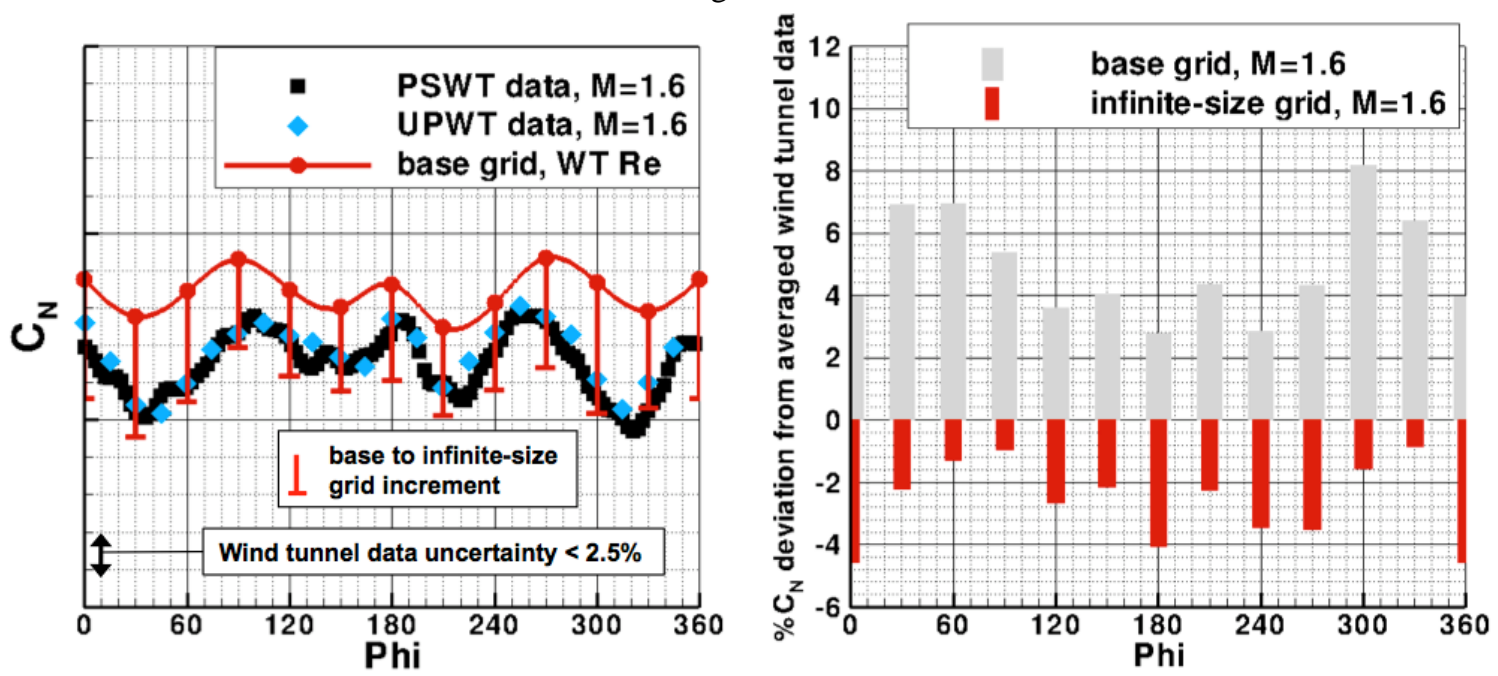

Figure 8. Computed $C_{N}$ from the base and infinite-size grid and correlation with wind tunnel data along with their percent deviation, $M=1.6, \alpha=8^{\circ}$.

Figure 9 presents the maximum deviation in the computed longitudinal aerodynamic coefficients from the WT data, across the examined roll angles using both the BG and IG, for various Mach numbers. The results based on the BG at the transonic Mach number indicated the highest deviation of roughly 23\% associated with the $C_{N}$ but improved to approximately $16 \%$ for the computations with the IG. However, the results at the supersonic flow conditions based on the BG showed a maximum deviation of only about $\pm 8 \%$ for all the computed coefficients with that level improving to approximately $\pm 5 \%$ for the IG. 


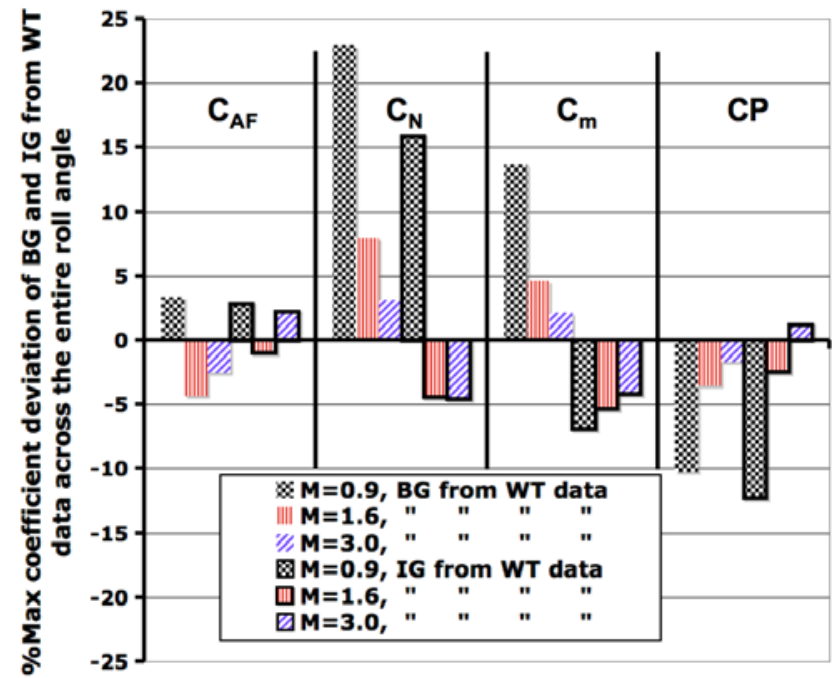

Figure 9. Percent maximum deviations in the computed longitudinal aerodynamic coefficients from the WT data across the examined roll angles using the BG and IG for various Mach numbers at $\alpha=\mathbf{8}^{\mathbf{0}}$.

\section{Computations at Flight Re}

The WT Re surface triangle definitions were employed to generate the corresponding volume grids for the flight Reynolds number computations. Table 2 shows the grid statistics and the computer resource requirements for FLT Re computations. Finer grid spacing was used primarily in the viscous layer to resolve the expected thinner boundary layer flow characteristic resulting from at least an order of magnitude higher Re than WT flow conditions. Such an effect caused an increase of about $20 \%$ to $30 \%$ more volume cells for the FLT Re cases than their WT Re counterpart grids. As expected, such an increase in the total number of cells, as well as additional number of iterations, resulted in about $50 \%$ more computer resource requirements for the FLT Re computations.

Table 2 ADAC-3 (A106) grid statistics and computer resource requirements for FLT Re cases

\begin{tabular}{|c|c|c|c|c|c|c|}
\hline Grid & $\begin{array}{c}\text { Number of } \\
\text { cells, } \boldsymbol{N}\end{array}$ & $\begin{array}{c}\mathbf{1}^{\text {st }} \text { cell height, } \\
\boldsymbol{\delta}_{\mathbf{1}} \text {, inches }\end{array}$ & $\begin{array}{c}\text { Number of } \\
\text { viscous cells }\end{array}$ & $\begin{array}{c}\text { Number of } \\
\text { surface } \\
\text { triangles }\end{array}$ & \#Iterations & $\begin{array}{c}\text { Computer } \\
\text { Resources } \\
\text { CPU-hr range }\end{array}$ \\
\hline coarse & $29,809,726$ & 0.0015 & $24,741,678$ & 377,272 & $18,000-30,000$ & $800-1,500$ \\
\hline base & $90,922,136$ & 0.001 & $66,390,036$ & $1,023,590$ & $18,000-30,000$ & $3,000-5,000$ \\
\hline fine & $267,733,262$ & 0.00066 & $165,583,035$ & $2,568,446$ & $18,000-30,000$ & $10,000-15,000$ \\
\hline
\end{tabular}

Complementary computations at FLT Re were performed at the selected flow conditions and the roll angles. Figure 10 shows the incremental effects in the computed $C_{A F}$ and $C_{N}$, due to Re obtained from the BG and the IG for $M=1.6$ and $\alpha=8^{\circ}$. Two general observations can be made from these results at these particular flow conditions. The first is that the computed coefficients at FLT Re were both lower than those obtained at WT Re across all roll angles. The second observation is that, surprisingly, the Re incremental effects were, for the most part, smaller than the ones computed because of the grid refinements. As a result, it can be concluded that the numerical predictions at the WT Re tend to provide more conservative estimates of these aerodynamic coefficients from a structural design standpoint for the vehicle than their counterparts at flight flow conditions. Therefore, these results suggest that future numerical activities, if constrained by computational resources, could mainly focus on the computations at WT Re with only limited and strategically chosen cases to address the incremental effects due to WT to FLT Reynolds numbers. 

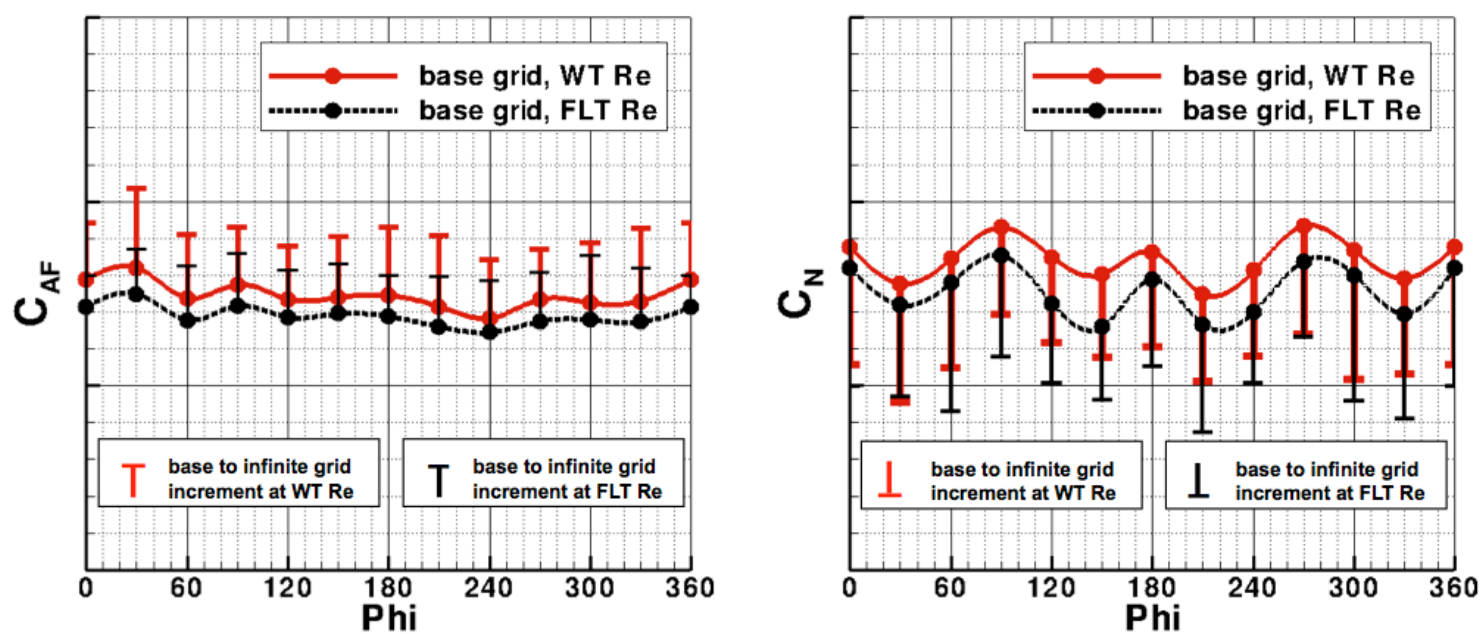

Figure 10. Computed $C_{A F}$ and $C_{N}$ from the base and infinite-size grid at WT and FLT Re, $M=1.6$,

$$
\alpha=\mathbf{8}^{\circ} \text {. }
$$

The bar graph in Figure 11 presents the computed results at the FLT Re along with those computed at the WT Re that were shown earlier in Figure 6. In general, the results in the longitudinal aerodynamic characteristics and the center of pressure over the examined flow conditions and roll angles indicated that the maximum error estimate from the BG to an IG were less than $\pm 10 \%$, with an exception. This exception is associated with the computed $C_{N}$ at $M=0.9$ and FLT Re that resulted in a maximum change of about $18 \%$ due to the grid refinement across the roll angles. This may suggest that the existing base grid was not sufficiently fine for resolving the transonic flow features correctly and thus causing an increase in the percent maximum change in the computed $\mathrm{C}_{\mathrm{N}}$.

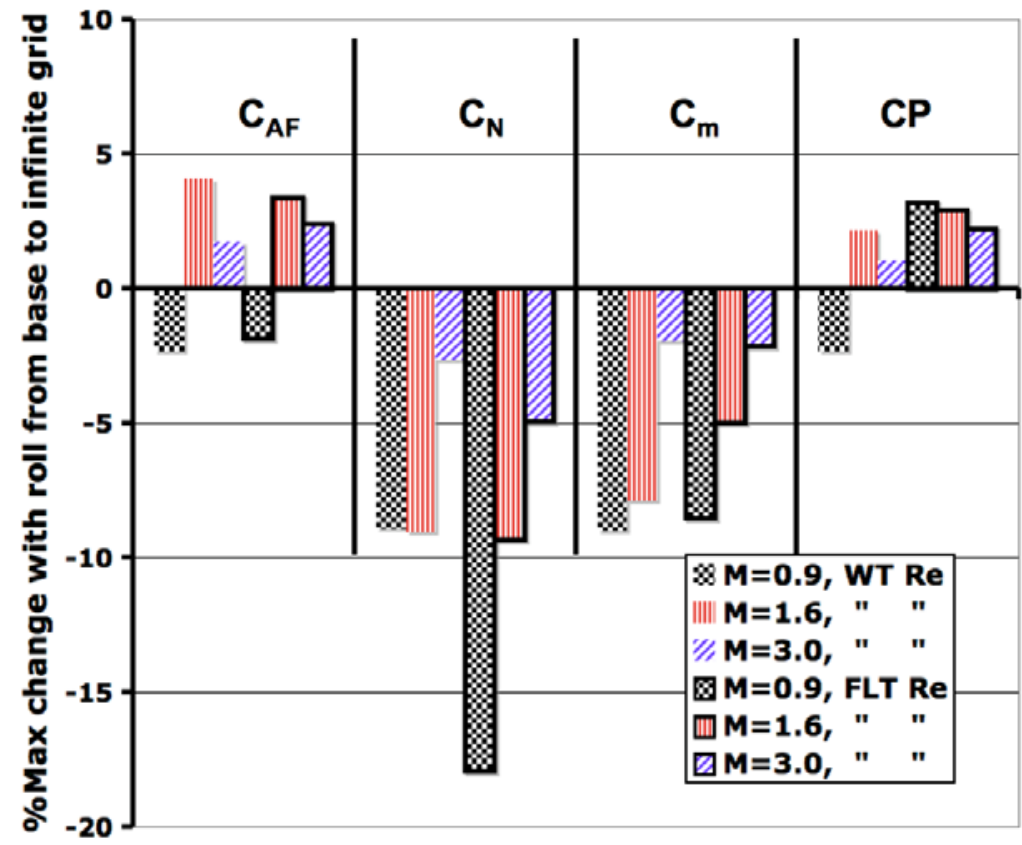

Figure 11. Percent maximum error estimate with roll angle in the computed longitudinal aerodynamic characteristics and the center of pressure from the base to an infinite-size grid for various Mach numbers at $\alpha=8^{\circ}$, WT and FLT Re.

\section{Concluding Remarks}


Numerical predictions for the longitudinal aerodynamic characteristics of the Ares I class of vehicles, based on the Reynolds averaged Navier-Stokes equations, are presented at flow conditions pertinent to the ascent phase of the flight trajectory. The results were obtained from the NASA Langley developed unstructured grid flow solver, USM3D, and were based on the Spalart-Allmaras turbulence model with an assumption that the flow was fully turbulent over the entire vehicle surfaces. The present analyses were performed at the selected flow conditions of $\alpha=8^{\circ}, M=0.9,1.6,3.0$ at both flight and wind tunnel Reynolds number. The focus of the analysis is to quantify the error estimates due to iterative convergence grid refinement in the computed aerodynamic coefficients of interest for the vehicle's last design cycle, designated as the A106 configuration. The validity of the approach to compute the associated error estimates, derived from a base grid to an extrapolated infinite-size grid, was first demonstrated on a sub-scaled wind tunnel model at representative flow conditions for which the experimental data existed. Such analysis at the transonic flow conditions revealed a maximum deviation of about $23 \%$ between the computed longitudinal aerodynamic coefficients with the base grid and the measured data across all the roll angles. This maximum deviation from the wind tunnel data was associated with the computed normal force coefficient at the transonic flow condition and was reduced to approximately $16 \%$ based on the infinite-size grid. However, all the computed aerodynamic coefficients with the base grid at the supersonic flow conditions showed a maximum deviation of only about $\pm 8 \%$ with that level being improved to approximately $\pm 5 \%$ for the infinite-size grid. A maximum error estimate of less than $\pm 10 \%$ was found, in the overall computed longitudinal aerodynamic characteristics at wind tunnel Reynolds numbers, between the base and the infinite-size grid across the examined roll angles and Mach numbers. In general, error estimate remained similar for the computations at flight Reynolds number with the exception of the computed normal force coefficient that was increased to approximately $18 \%$ at the transonic Mach number.

\section{Acknowledgement}

The authors would like to thank Edward B. Parlette of ViGYAN, Inc. for generating the unstructured grids used in the present computational analysis.

\section{References}

1. NASA’s Exploration Systems Architecture Study, NASA-TM-2005-214062, Nov. 2005.

2. Frink, N. T., Pirzadeh, S. Z., Pandya, M. J., Bhat M. K. “The NASA Tetrahedral Unstructured Software System,” The Aeronautical Journal, Vol. 104, No. 1040, October 2000, pp. 491-499.

3. Alexandrov, N. M., Atkins, H. L., Bibb, K. L., Biedron R. T., Carpenter, M. H., Gnoffo, P. A., Hammond, D. P., Jones, W. T., Kleb, W. L., Lee-Rausch, E. M., Nielsen, E. J., Park, M. A., Raman, V. V., Roberts, T. W., Thomas, J. L., Vatsa, V. N., Viken, S. A., White, J. A., Wood, W. A., "Team Software Development for Aerothermodynamic and Aerodynamic Analysis and Design,” NASA TM-2003-212421, November 2003.

4. Buning, P. G., Chan, W. M., Renze, K. J., Sondak, D. L., Chiu, I. T., and Slotnick, J. P., OVERFLOW User's Manual, Version 1.6ab, 26 January 1993, NASA Ames Research Center, Moffett Field, CA, Jan. 1993.

5. Abdol-Hamid, K. S., Ghaffari, F., and Parlette, E. B., "Overview of Ares I CFD Ascent Aerodynamic Data Development and Analysis Based on USM3D,” AIAA Paper No. 2011-0015.

6. Spalart, P. R., and Allmaras, S. R., A One-Equation Turbulence Model for Aerodynamic Flows. AIAA Paper No. 1992-0439.

7. Jones, W. P. and Launder, B. E., “The Prediction of Laminarization With a Two-Equation Model of Turbulence,” Int. J. Heat \& Mass Transf., Vol. 15, No. 2, Feb. 1972, pp. 301-314.

8. Menter, F. R., “Improved Two-Equation k-omega Turbulence Models for Aerodynamic Flows,” NASA TM-103975, October 1992.

9. Samareh, J. A., "GridTool: A Surface Modeling and Grid Generation Tool, Proceedings of the Workshop on Surface Modeling, Grid Generation, and Related Issues in CFD Solutions,” NASA Lewis Research Center, Cleveland, OH, NASA CP-3291, 1995, May 9-11, 1995.

10. Pirzadeh, S. Z., “Advanced Unstructured Grid Generation for Complex Aerodynamics Applications,” AIAA Paper No. 2008-7178, August 2008.

11. 4th AIAA CFD Drag Prediction Workshop (DPW), http://aaac.larc.nasa.gov/tsab/cfdlarc/aiaa-dpw/ [cited 28 June 2009].

12. $1^{\text {st }}$ AIAA CFD High Lift Prediction Workshop (HiLiftPW), http://hiliftpw.larc.nasa.gov [cited 28 June 2010].

13. Cavallo, P. and Grismer, M., “A Parallel Adaptation Package For Three-Dimensional Mixed-Element Unstructured Meshes,” Journal of Aerospace Computing, Information, and Communication 2005, 1542-9423 Vol. 2, No. 11, (433451) doi: 10.2514/1.16220.

14. Erickson, G. E. and Wilcox, F. J., “Ares I Aerodynamic Testing at the NASA Langley Unitary Plan Wind Tunnel,” AIAA Paper No. 2011-0999. 
15. Pinier, J. T., Niskey, C. J., Hanke, J. L., and Tomek, W. G., “Ares I Aerodynamic Testing at the Boeing Polysonic Wind Tunnel," AIAA Paper No. 2011-0998.

16. Houlden, H.P., Favaregh, A.L., and Hemsch, M.J., "Quantification of the Uncertainties for the Ares I A106 Ascent Aerodynamic Database,” AIAA Paper No. 2010-4926. 